DESY $97-070$

April 1997

Series Tests of Fine Mesh Photomultiplier Tubes in Magnetic Fields of up to 1.2 Tesla

H1 SpaCal Group

ISSN 0418-9833 


\title{
Series Tests of Fine Mesh Photomultiplier Tubes in Magnetic Fields of up to 1.2 Tesla
}

\author{
The H1 SpaCal Group
}

April 2, 1997

\begin{abstract}
The new lead/scintillating-fibre calorimeter ("SpaCal") for the backward region of the $\mathrm{H} 1$ experiment at HERA (DESY) is equipped with fine mesh phototubes which operate in a magnetic field close to $1 \mathrm{~T}$. A large sample of these tubes of the types Hamamatsu R5505 and R5506, and Hamamatsu R2490-06, have been tested in fields of up to $1.2 \mathrm{~T}$. We have investigated the cathode homogeneity with and without magnetic field, the gain loss under the influence of the magnetic field, and stability with time. For a subsample of tubes, we have performed additional studies on stability with respect to temperature changes, variation of gain as a function of the magnetic field, high voltage discharges, single photo-electron response, and linearity. We finally summarize the experience with these tubes after one year of operation in the experiment.
\end{abstract}

R.-D. Appuhn ${ }^{4}$, C. Arndt ${ }^{4}$, E. Barrelet ${ }^{10}$, R. Barschke, U. Bassler ${ }^{10}$, T. Bipp ${ }^{4}$, V. Boudry ${ }^{9}$, F. Brasse ${ }^{4}$, D. Bruncko ${ }^{6}$, R. Buchholz ${ }^{4}$, S. Chechelnitski ${ }^{8}$ B. Claxton ${ }^{14}$, G. Cozzika ${ }^{3}$, J. Cvach ${ }^{11}$, S. Dagoret-Campagne ${ }^{10}$, W.D. Dau ${ }^{15}$, H. Deckers ${ }^{2}$, T. Deckers ${ }^{2}$, F. Descamps ${ }^{10}$, M. Dirkmann ${ }^{2}$, J. Dowdell ${ }^{14}$, V. Efremenko ${ }^{8}$, E. Eisenhandler ${ }^{7}$, A.N. Eliseev ${ }^{17}$, G. Falley ${ }^{4}$, J. Ferencei ${ }^{6}$, M. Fleischer ${ }^{2}$, B. Fominykh ${ }^{8}$, K. Gadow ${ }^{4}$, U. Goerlach ${ }^{4}$, L.A. Gorbov ${ }^{17}$, I. Gorelov ${ }^{8}$, M. Grewe ${ }^{2}$, L. Hajduk ${ }^{18}$, A. Heck ${ }^{5}$, I. Herynek ${ }^{11}$, J. Hladký ${ }^{11}$, M. Hütte' ${ }^{2}$, H. Hutter ${ }^{2}$, W. Janczur ${ }^{13}$, J. Janoth ${ }^{5}$, L. Jönsson ${ }^{16}$, H. Kolanoski ${ }^{2}$, V. Korbel ${ }^{4}$, F. Kriváñ ${ }^{6}$, D. Lacour $^{10}$, B. Laforge ${ }^{3}$, F. Lamarche ${ }^{9}$, M.P.J. Landon ${ }^{7}$, J.-F. Laporte ${ }^{3}$, F. Lehner ${ }^{4}$, R. Maraček ${ }^{6}$, K. Meier ${ }^{5}$, A. Meyer ${ }^{4}$, A. Migliori ${ }^{9}$, F. Moreau ${ }^{9}$, G. Müller ${ }^{4}$, P. Murín ${ }^{6}$, V. Nagovizin ${ }^{8}$, T.C. Nicholls ${ }^{1}$, D. Ozerov ${ }^{8}$, E. Perez ${ }^{8}$, J.P. Pharabod ${ }^{9}$, R. Pöschl ${ }^{2}$, A. Rostovtsev ${ }^{8}$, C. Royon ${ }^{3}$, K. Rybicki ${ }^{13}$, R. Schediwy ${ }^{5}$, S. Schleif ${ }^{5}$, K. Schmitt ${ }^{5}$, A. Schuhmacher ${ }^{2}$, A. Semenov ${ }^{8}$, V. Shekelyan ${ }^{8}$, Y. Sirois ${ }^{9}$, P.A. Smirnov ${ }^{17, c}$, V. Solochenko ${ }^{8}$, J. Śpalek ${ }^{6}$, S. Spielmann ${ }^{\theta}$, H. Steiner ${ }^{4, \alpha}$, A. Stellberger ${ }^{5}$, J. Stiewe ${ }^{5}$, M. Taševský ${ }^{12}$, V. Tchernyshov ${ }^{8}$, K. Thiele ${ }^{4}$, E. Tzamariudaki ${ }^{4}$, S. Valkár ${ }^{12}$, D. VanDenPlas ${ }^{8}$, G. Villet ${ }^{3}$, K. Wacker ${ }^{2}$, A. Walther ${ }^{2}$, M. Weber ${ }^{5}$, D. Wegener ${ }^{2}$, T. Wenk ${ }^{2}$, J. Žáček ${ }^{12}$, A. Zhokin ${ }^{8}$, and K. Zuber ${ }^{5}$

${ }^{1}$ School of Physics and Space Research, University of Birmingham, Birmingham, UK ${ }^{a}$

${ }^{2}$ Institut für Physik, Universität Dortmund, Dortmund, Germany ${ }^{b}$

${ }^{3}$ CEA, DSM/DAPNIA, CE-Saclay, Gif-sur-Yvette, France

${ }^{4}$ DESY, Hamburg, Germany ${ }^{b}$ 
${ }^{5}$ Institut für Hochenergiephysik, Universität Heidelberg, Heidelberg, Germany ${ }^{b}$

${ }^{6}$ Institute of Experimental Physics, Slovak Academy of Sciences, Košice, Slovak Republic ${ }^{c, d}$

${ }^{7}$ Queen Mary and Westfield College, London, UK ${ }^{a}$

${ }^{8}$ Institute for Theoretical and Experimental Physics, Moscow, Russia ${ }^{e}$

${ }^{\theta}$ LPNHE, Ecole Polytechnique, IN2P3-CNRS, Palaiseau, France

${ }^{10}$ LPNHE, Universités Paris VI and VII, IN2P3-CNRS, Paris, France

${ }^{11}$ Institute of Physics, Czech Academy of Sciences, Prague, Czech Republic ${ }^{c, f}$

12 Nuclear Center, Charles University, Prague, Czech Republic ${ }^{c, f}$

${ }^{13}$ Insitute for Nuclear Physics, Cracow, Poland ${ }^{g}$

${ }^{14}$ Electronics Division, Rutherford Appleton Laboratory, Chilton, Didcot, UK ${ }^{a}$

${ }^{15}$ Institut für Reine und Angewandte Kernphysik, Universität Kiel, Kiel, Germany ${ }^{b}$

${ }^{16}$ Physics Department, University of Lund, Sweden ${ }^{h}$

${ }^{17}$ Lebedev Physical Institute, Moscow, Russia

a Supported by the UK Particle Physics and Astronomy Research Council, and formerly by the UK Science and Engineering Research Council

b Supported by the Bundesministerium für Forschung und Technologie, FRG under contract numbers 6DO57I, 6HH27I, 6HD27I and 6KI17P

' Supported by the Deutsche Forschungsgemeinschaft

d Supported by VEGA SR grant no. 2/1325/96

- Supported by INTAS-International Association for the Promotion of Cooperation with Scientists from Independent States of the Former Soviet Union. Co-operation Agreement INTAS-930044 .

f Supported by GA $\check{C} R$, grant no. 202/93/2423, GA AV $\check{C} R$, grant no. 19095 and GA UK, grant no. 342

S Supported by the Polish State Committee for Scientific Research, grant No. 204209101

${ }^{h}$ Supported by the Swedish Natural Science Council

a Permanent address: Lawrence Berkeley Laboratory, University of California, Berkeley, California 94720

\section{Introduction}

The storage ring HERA at DESY where positrons of $27.5 \mathrm{GeV}$ collide with protons of $820 \mathrm{GeV}$ is devoted to the investigation of deep inelastic scattering (DIS) processes, with the main emphasis on the high $Q^{2}$ regime. Recently, the low $x_{B j o r k e n}$ region attracted growing interest which led to the re-design of the "backward" (with respect to the proton direction) region of the H1 detector. In order to ensure a precise measurement of the scattered positron's energy and momentum vector, the old backward electromagnetic calorimeter BEMC [1], a coarsely segmented lead/scintillator sandwich calorimeter, was replaced by a new finely segmented lead/scintillatingfibre calorimeter ("SpaCal") consisting of an electromagnetic and a hadronic section which covers a larger angular range than the BEMC [2]. In addition, the former Backward Proportional Chamber (BPC) was replaced by a Backward Drift Chamber (BDC). While the BEMC was read out via photodiodes, the new calorimeter uses photomultipliers so that the noise level is much lower and the timing resolution is considerably improved.

The new calorimeter covers the polar angular range from $153^{\circ}$ to $177.5^{\circ}$. The polar angle is measured w.r.t. the proton beam direction so that large polar angles indicate small physical scattering angles of the positron. 
The electromagnetic section of the SpaCal [2] is 28 radiation lengths deep. It is segmented into 1192 cells with a cross section of $40.5 \cdot 40.5 \mathrm{~mm}^{2}$. Each cell is read out by a 1 " photomultiplier. The scintillating fibres exiting a single calorimeter module are bundled and glued onto a plexiglass light mixer which in turn is glued onto the cathode window of the phototube. Two cells form a "submodule" which is the smallest construction unit of the calorimeter. Eight submodules form one square shaped "supermodule"; the supermodules form the logical units of the SpaCal.

The hadronic section [3], placed behind the electromagnetic one, is 1.1 nuclear interaction lengths deep. Its segmentation is coarser: it consists of 128 cells with a cross section of 119.3 . $119 \mathrm{~mm}^{2}$. This choice of cross section is matched to the transverse size of hadronic showers. Each cell is read out by a 2" phototube.

Using phototubes for the light readout circumvents the noise problem connected with the use of photodiodes and their preamplifier. The detection of minimum ionizing particles (MIPs) becomes possible, and the separation of electromagnetically interacting particles from hadrons is significantly improved. For operation in high magnetic fields, however, there exists only a limited choice of tubes. We have chosen Hamamatsu phototubes with "fine mesh" dynodes oriented perpendicular to the tube axis.

The paper is organized as follows: after a description of the fine mesh phototubes, we will describe the experimental setups for test measurements with and without magnetic field and discuss their results.

Based on investigations made with a selection of tubes, we will further discuss some special aspects such as the influence of temperature changes, the variation of gain as a function of the magnetic field, the occurrence of high voltage discharge effects ("sparking"), the single photoelectron response, and linearity for various operating currents. We will finally review the first year of running in SpaCal under real operating conditions. 


\section{Hamamatsu "Fine Mesh" Phototubes}

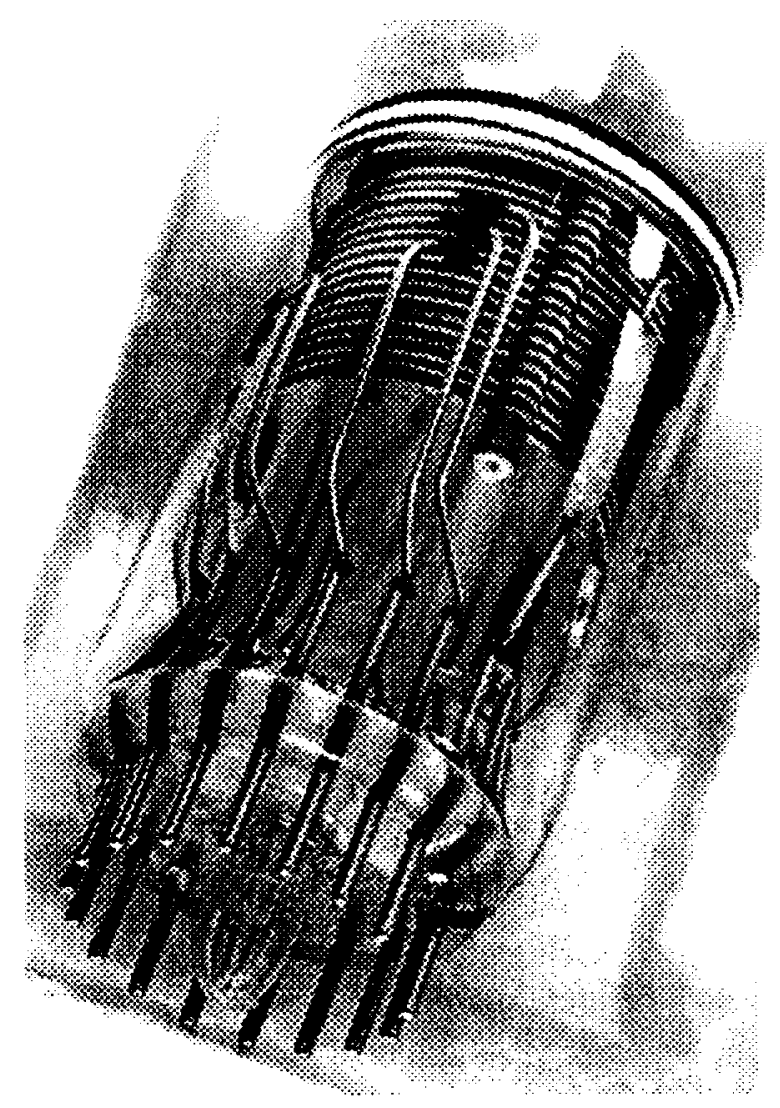

Figure 1: Photograph of a Hamamatsu R5505 fine mesh phototube, showing the typical parallel arrangement of the dynodes.

Specially constructed phototubes are needed for operation in high magnetic field environments. A suitable choice is the "proximity fine mesh dynode" tube from Hamamatsu Photonics [4]. Its main feature is the tight arrangement of dynodes which consist of fine gratings with "meshes": a stack of 15 or 16 dynodes with an inter-dynode distance of roughly half a millimeter are oriented perpendicular to the tube axis. If the tube is aligned with the magnetic field, secondary electrons emitted from a dynode stage and accelerated towards the next one, drift with no or only a small velocity component perpendicular to the magnetic field. Thus, Lorentz forces are low and cause only a limited perturbation of electron trajectories which maintains a substantial gain even in strong magnetic fields. The origin of gain variations as a function of the magnetic field applied is dicussed in [5] .

Due to the grid structure, some of the electrons accelerated between mesh dynodes get lost before they can reach the anode. This can be described by a geometrical transmission factor $\alpha$ of the tubes which is about 0.45 . This value follows roughly from the geometrical conditions: the grid bar structure has a width of $6 \mu \mathrm{m}$ while the mesh hole diameter is $12 \mu \mathrm{m}$. The parameter $\alpha$ influences the voltage dependence of the phototube's gain which is of the form

$$
G(U) \propto\left(U-U_{0}\right)^{\alpha n} \text {. }
$$

Here, $\mathrm{G}$ is the gain as a function of the voltage $U ; U_{0}$ is a parameter close to $0 \mathrm{~V}$, and $\mathrm{n}$ is the number of dynode stages [7]. 
The phototubes chosen for the SpaCal electromagnetic section are the Hamamatsu R5505 and R5506 tubes. They have a cathode diameter of roughly $1 "(2.54 \mathrm{~cm})$ and will be labellel 1 " tubes. The tubes for the hadronic section are of the type Hamamatsu R2490-06. They have a cathode diameter of about $2 "(5.08 \mathrm{~cm})$ and will be labelled 2" tubes. The R5506 tube has a UV hard instead of a boron silicate window which the R5505 tube has; it is less sensitive to radiation and therefore installed in the region close to the beam pipe which is exposed to higher radiation doses. Throughout the paper, we will refer to both types as R5505. Cathode and dynode materials are of the bialkali type. According to the manufacturer's data sheets, the typical high voltages range to $2300 \mathrm{~V}$. At $\mathrm{H} 1$, the tubes are operated at voltages between 1600 $\mathrm{V}$ and $2000 \mathrm{~V}$. In the tests described below, the performance of $1390 \mathrm{R} 5505$ and 144 R2490-06 tubes has been investigated.

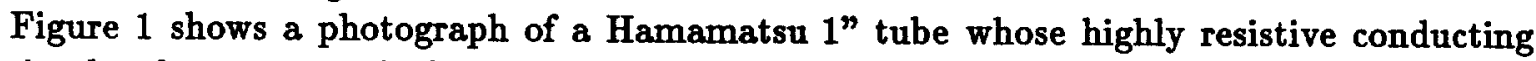
coating has been removed. Clearly visible is the parallel arrangement of the dynodes, perpendicular to the tube axis. The characteristic features of the fine mesh tubes discussed in this paper are listed in table 1.

\begin{tabular}{|l|c|c|}
\hline & R 5505 & R 2490-06 \\
\hline \hline Length & $(40.0 \pm 1.5) \mathrm{mm}$ & $(50.0 \pm 2.0) \mathrm{mm}$ \\
\hline Outer diameter & $(25.8 \pm 0.7) \mathrm{mm}$ & $(52.0 \pm 1.0) \mathrm{mm}$ \\
\hline Effective diameter of cathode at 0 T & $17.5 \mathrm{~mm}$ & $36.0 \mathrm{~mm}$ \\
\hline number of dynodes & 15 & 16 \\
\hline Gain at 0 T & $5 \cdot 10^{5}$ & $10^{7}$ \\
\hline Dark current & $5 \mathrm{nA}$ & $10 \mathrm{nA}$ \\
\hline
\end{tabular}

Table 1: Survey of fine mesh phototube parameters 


\section{Description of the Test Setup}

\subsection{Setup for Tests without Magnetic Field}

The general arrangement for measurements without magnetic field is such that the tubes to be tested are mounted in a matrix which can be scanned over by a light source with the help of two step motors. The matrix is made of aluminium and accomodates $8 \times 81$ " tubes with their faces in parallel to the matrix front plane. On top of the matrix, an additional set of four 2" tubes can be mounted. The entire setup is housed inside a light tight wooden box.

The two step motors, for movements in the horizontal and vertical directions, are mounted in parallel to the aluminium matrix and thus allow a movement in $x$ and $y$ across the PM cathodes. The entire range of a single motor is $300 \mathrm{~mm}$ in steps of $0.001 \mathrm{~mm}$, with a positioning accuracy of $0.003 \mathrm{~mm}$. The range of the step motors allows the scan of 641 "tubes [2": 4] in one measurement cycle. (We will use the square bracket notation throughout the paper to indicate corresponding quantities for the 2 " tubes.) During the test measurements the orientation in space of all tubes is kept fixed.

The two light sources carried by the step motors illuminate the phototubes via two plastic fibres with diameters of $1 \mathrm{~mm}$. One fibre is positioned at a distance of $10 \mathrm{~mm}$ from the cathode in order to illuminate its entire surface. The second one is located close to the cathode surface at a distance of only $0.2 \mathrm{~mm}$ in order to illuminate a single spot on the cathode surface with a diameter of about $1.5 \mathrm{~mm}$. The first one serves to calibrate the tube, the second one is used for a cathode homogeneity scan.

Each fibre is connected to its own light emitting diode (LED), and in turn, each LED feeds its light into three fibres: The scanning/calibrating fibre, and two fibres, each illuminating a reference tube (Philips AVP 150 and Philips SRC 75 B01). The LEDs are fired by a specially developed pulser system. The two light pulser systems are triggered by pulses generated via a CAMAC output register. The LED pulses have a triangular shape with $10 \mathrm{~ns}$ rise and $40 \mathrm{~ns}$ fall time.

High voltage is applied to the phototubes by a LeCroy HV4032A device which is controlled via an interface and programmed via the CAMAC system. Voltages for individual channels were equalized to a precision of better than $\pm 1 \%$. Readout of the phototubes is performed by a LeCroy 2249 ADC.

The measuring cycle is performed as follows: after a warm-up time of one hour at $2000 \mathrm{~V}$, the high voltages applied to all tubes are varied in order to determine the HV dependence of the gains. This is done in steps of $10 \mathrm{~V}$, starting at $1500 \mathrm{~V}$ for the 1 " tubes [2": $1200 \mathrm{~V}$ ]. At each HV value, a pedestal determination of the ADC is made with 100 "empty" pulses, i.e. without illuminating the phototube, followed by a second run with 100 light pulses onto the cathode. The resulting mean ADC signal has an accuracy of better than $1 \%$. The cycle is stopped when the ADC shows an overflow, or when the high voltage reaches its limit of $2300 \mathrm{~V}$. This procedure delivers an individual "calibration voltage" for each phototube which is defined as the voltage where its $A D C$ value equals that of the reference tubes within a few percent.

After determination of the calibration voltage, a position scan over the cathode surface of the tube is performed in steps of $0.5 \mathrm{~mm}$, with 100 light pulses per spot. Another pedestal run as described above precedes the scan. For the 1" tubes, the scan illuminates a square array of $49 \times 49$ spots [2": $60 \times 60$ ]. This corresponds to roughly 1000 spots covering the sensitive area of a 1" cathode. The results of two such scans, one for a nicely homogeneous and one for a rather non - homogeneous tube, are shown in Figure 2.

After the homogeneity scan, the tubes are tested for stability at their calibration voltages. 
They are illuminated every 30 minutes. This is done 72 times (R5505) [2": 20 times]. The total time needed for a complete testing cycle is about 5 days [2": $12 \mathrm{~h}$ ].
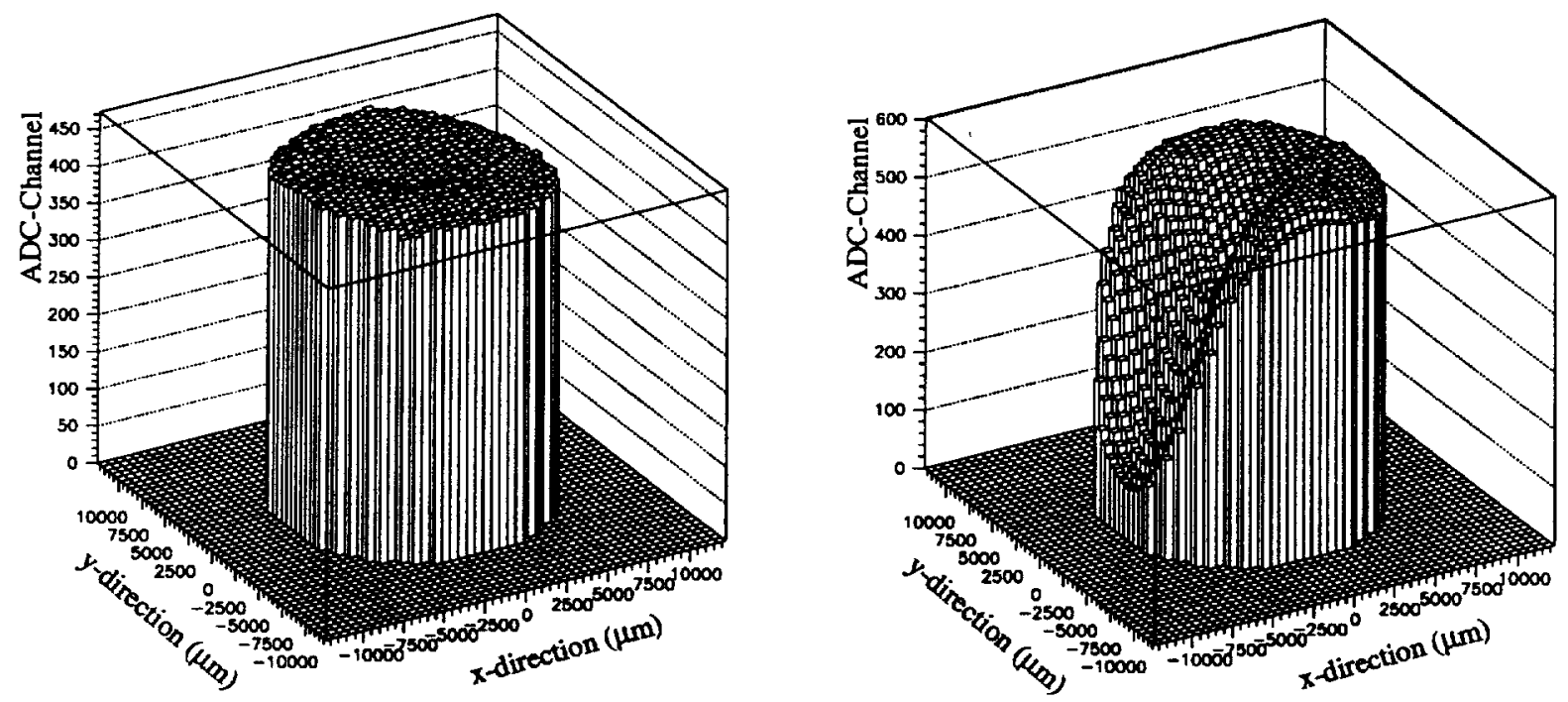

Figure 2: Cathode scan profiles of two 1" tubes, with step size of $0.5 \mathrm{~mm}$. The plots show the pedestal corrected $A D C$ values as a function of the scan position. Left: uniform cathode. Right: cathode with high degree of inhomogeneity. One hundred pulses were taken per point. 

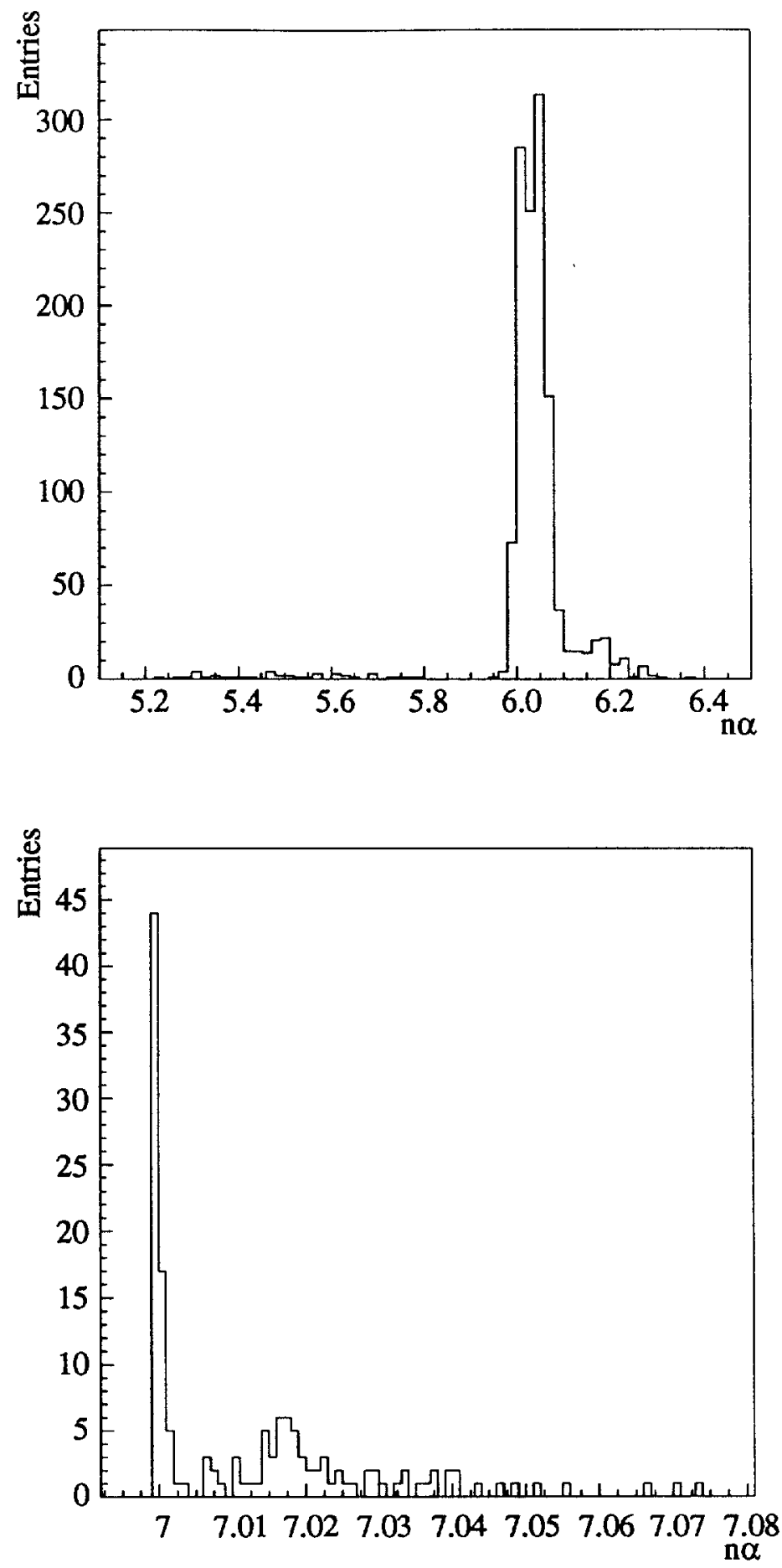

Figure 3: Distribution of exponents an for the high voltage dependence of the gain, derived from a power law fit (see text). Top: Distribution for the 1" tubes. Bottom: Distribution for the 2" tubes 


\subsection{Setup for Tests in a Magnetic Field}

The performance of the phototubes under the influence of a strong magnetic field was tested at DESY with the help of a large dipole magnet (Oerlikon MA 1-11) which provides a homogeneous field of up to $2 \mathrm{~T}$, with a precision of $\pm 0.001 \mathrm{~T}$. The space available for tests is about $200 \mathrm{~cm}$ in length, $48.3 \mathrm{~cm}$ in width, and $16.8 \mathrm{~cm}$ in height. The field direction is vertical, the axes of the tubes to be tested have to be aligned with the field.

The setup used for the field tests of the 1" tubes is a matrix of 32 fixed voltage dividers with four rows and eight columns, housed in a light tight brass box so that 32 tubes can be tested at a time. Ilumination of the tubes is done via a movable aluminium sleigh which carries the light fibres. In this way, one row containing four tubes can be tested at a time. For the next measurement, the sleigh is moved manually to the neighbouring row of phototubes as electrical step motors cannot be operated in such high fields. Accurate positioning (within $0.1 \mathrm{~mm}$ ) is guaranteed through a precise mechanical locking device.

Each phototube in a row of four tubes can be illuminated via a fibre bundle which contains 25 fibres with a diameter of $0.5 \mathrm{~mm}$. The 25 fibres are mounted in a fixed arrangement of three concentric circles around one central fibre fitting onto the cathode. The diameter of the outer circle is $15 \mathrm{~mm}$ which corresponds to the guaranteed effective cathode diameter for the R5505 tube at $1.2 \mathrm{~T}$. The fibres are fed by 25 LED pulsers similar to those described above. Each pulser drives five fibres: four of them are connected to four corresponding spots at the illumination masks which are carried by the sleigh. The fifth one is guided to the cathode of a reference tube (Philips SRC 75 B01) outside the field. In total, there are $25 \times 4$ fibres fed to the sleigh, plus 25 fibres fed to the reference tube.

The light from the LED is coupled to the fibres via a small piece cut from a $1.5 \mathrm{~mm}$ diameter fibre which is glued to the LED. It works as a light mixer and distributes the blue LED light as homogeneously as possible to the five fibres which are glued to its other end. During the glueing procedure, the light output of each individual fibre was monitored by a photodiode. The outputs were found to be roughly the same within a factor of two.

The phototubes are read out by a LeCroy 2249 ADC. To accommodate the expected large differences in pulse height from measurements with and without a magnetic field, we apply a calibrated $39 \mathrm{~dB}$ attenuator (resulting in an attenuation factor of 90 with a $3 \%$ accuracy).

The pulsers are triggered via a CAMAC output register. The high voltage is delivered by a programmable four channel CAEN $470 \mathrm{~N}$ power supply which is controlled by a CAMAC controller via a CAEN interface.

The testing procedure is similar to that one with no. magnetic field: first, a calibration run is made by firing successively all 25 LEDs ( 100 pulses per spot, and pedestal measurement for each LED), then the averages of the pedestal corrected ADC values are stored. The cycle starts at $1400 \mathrm{~V}$ [2": $1220 \mathrm{~V}$ ] and proceeds in steps of $30 \mathrm{~V}$. It is stopped when the ADC shows an overflow, or the high voltage reaches $2300 \mathrm{~V}$. For the homogeneity scans 100 pulses per spot are fired, for each of the 25 spots on a 1 " tube cathode. Three measurement cycles are performed at fields of $0 \mathrm{~T}, 0.95 \mathrm{~T}$ [2": $0.88 \mathrm{~T}$ ], and 1.2 T. Testing one set of $4 \times 81^{\prime \prime}$ tubes takes 3 hours. Testing one 2 " tube requires 10 minutes.

The setup used for the 2 " tubes is much simpler. It consists of a light tight aluminium box containing a voltage divider. One blue LED illuminates the entire cathode and the reference tube. No homogeneity scan is made. The readout is identical to that one used for the 1 " tubes. 

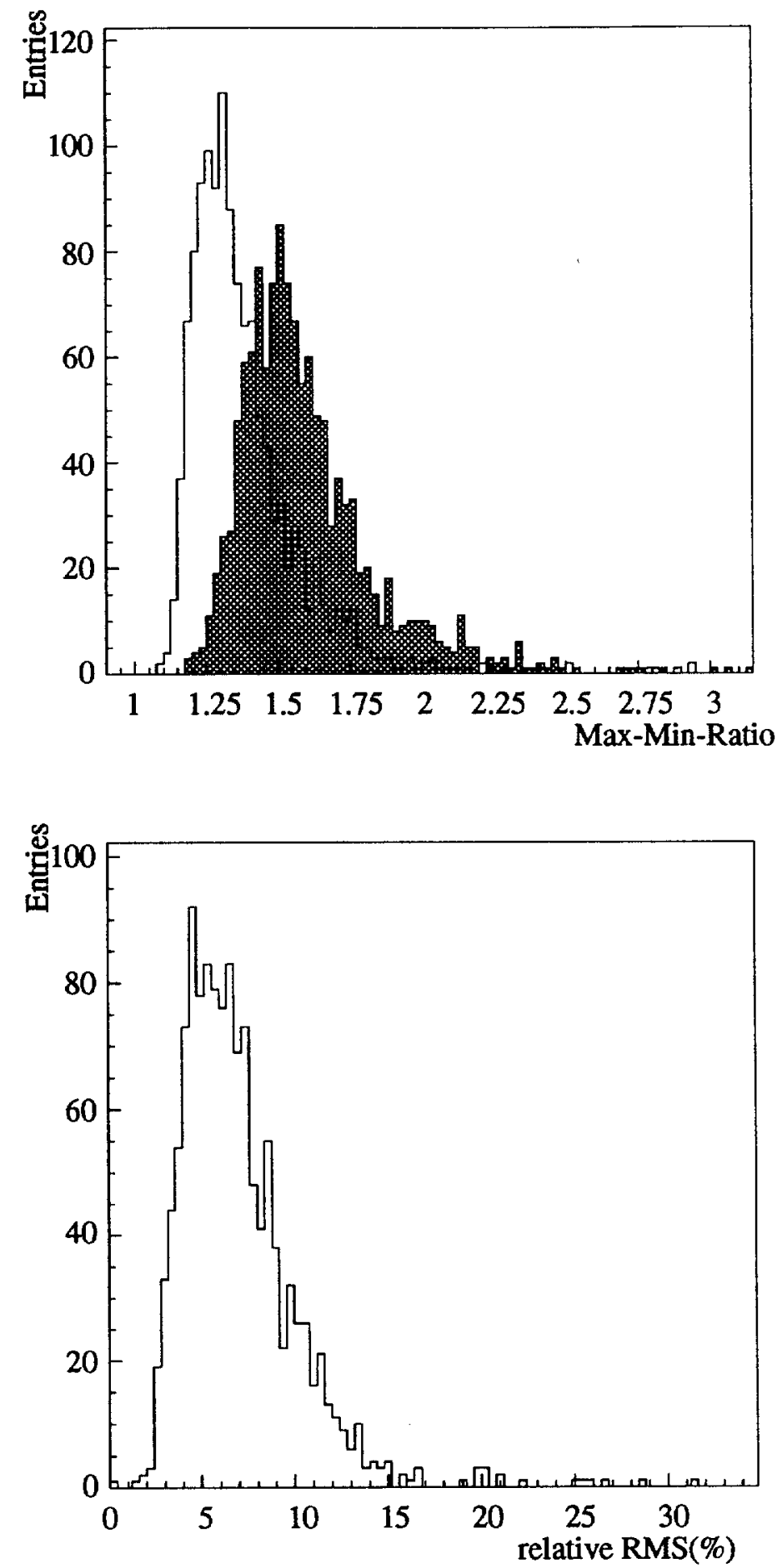

Figure 4: Top: Max-to-min ratio of the cathode response for the 1 " tubes without field and at 1.2 $T$ (hatched area). One observes a shift towards higher inhomogeneities. Bottom: Distribution of relative response fluctuations (r.m.s. divided by mean). Each entry is based on all data points from an individual cathode scan without magnetic field. 

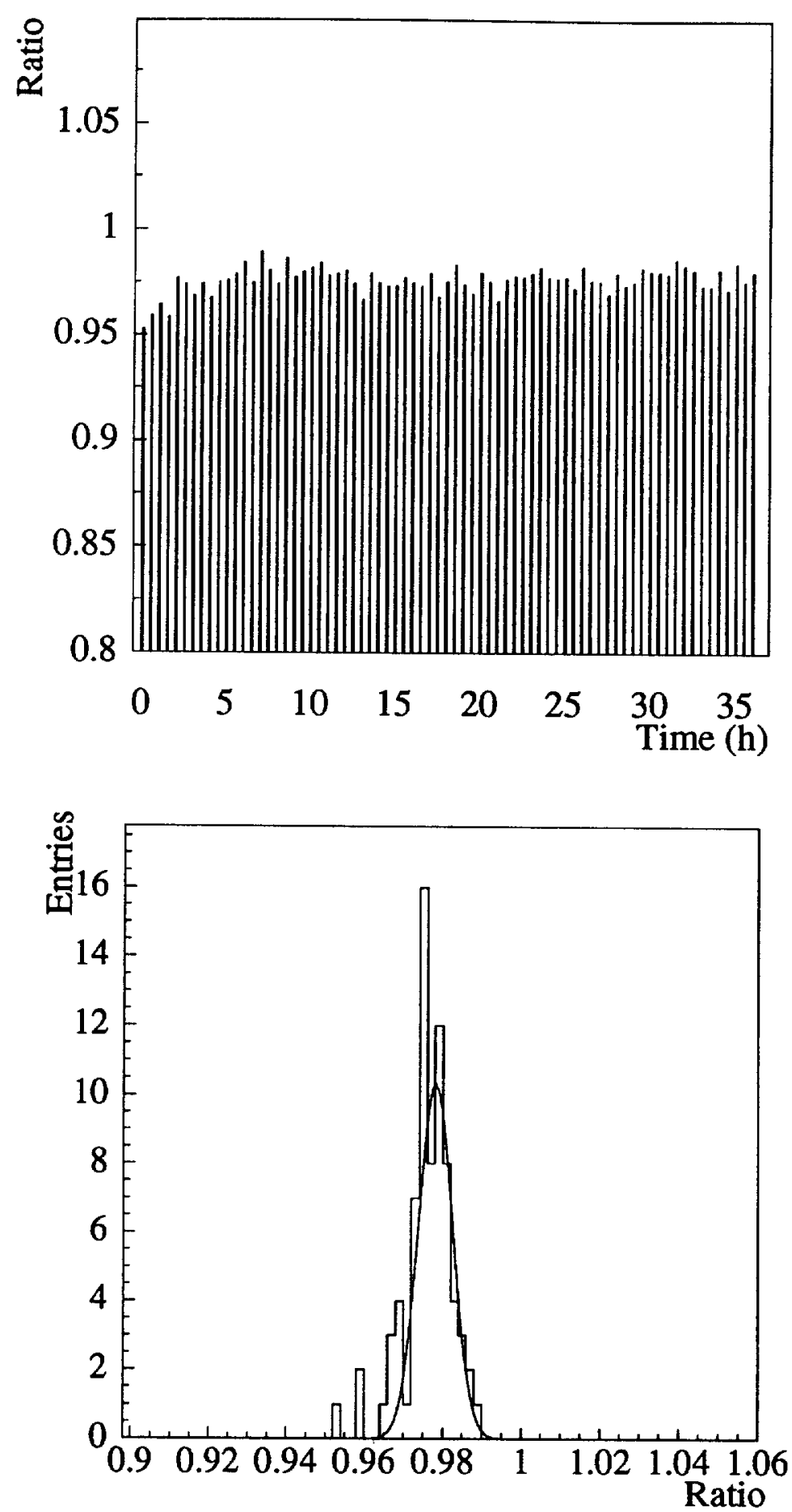

Figure 5: Example for a stability measurement. Top: Ratio of responses of a 1" tube and the reference tube as a function of time. The small initial rise is due to warm-up effects. Bottom: Projection of the ratios, and the result of a Gaussian fit to determine the degree of fluctuation. 


\section{Test Results}

Using the setups described above, a total of 1390 phototubes of the type R5505, and of 144 tubes of the type R2490-06, have been subjected to a series of test measurements: high voltage dependence of gains, homogeneity of cathode response, stability with time, dark current, and change of gain and cathode homogeneity under the influence of a strong magnetic field. In the following, we will discuss the results of these investigations in some detail.

\subsection{Results of Tests with No Magnetic Field}

For each individual tube a calibration measurement was performed in which the amplitude $S$ of the output signal as a function of the high voltage is determined. The shape of this curve which reflects the dependence of gain on the high voltage $U$ is parametrized by the expression

$$
S=\left(\frac{U-U_{0}}{A}\right)^{\alpha n}
$$

where $S$ means ADC counts, $U_{0}$ is a parameter close to $0 \mathrm{~V}, n$ is the number of dynodes, and $\alpha$ denotes a factor depending mainly on the shape and geometrical arrangement of the dynodes (see Section 2). $A$ is a normalization factor.

The mean value of the exponent $\alpha$ nas found to be 6.1 for the 1 " tubes, and 7.0 for the 2 " tubes (Figure 3 ). The slightly larger value for the 2 " tubes can be explained by the additional (16th) dynode in that type, together with a somewhat larger geometrical transmission factor which is of the order of 0.4 for the 1 " tubes. (It should be noted that the parameter $\alpha$ for phototubes with the standard linear focusing arrangement of dynodes is around $0.7-0.8$.)

The homogeneity of the photocathode response can be characterized by two quantities: the ratio of the values of highest and lowest response, the so-called max-to-min ratio, and the (relative) standard deviation of all ADC values available for an individual cathode. The distributions of these parameters are shown in Figure 4, top and bottom. The mean value of the max-to-min distribution is about 1.4 (top). Thirty tubes out of 1390 show a max-to-min ratio larger than 2.5 [2": 12 out of 144] and have been rejected. For an ideally homogeneous cathode, the value r.m.s./mean should be zero. The mean of this ratio for the R5505 tubes tested amounts to $7 \%$ (Figure 4 , bottom ) [2": $13 \%$ ].

From the stability measurements, two quantities are extracted: first, the ratio of signals of the Hamamatsu tube to be tested to those of the reference tube is determined as a function of time. A typical behaviour is shown in Figure 5 (top). By fitting a linear curve we define a drift parameter as the slope of this curve. According to this procedure only $0.9 \%$ of the tubes show a drift of more than $0.2 \%$ per hour. It should be mentioned that the errors on the slope parameters fit are generally larger than the slope values themselves, i.e. the tubes do not exhibit significant drifts during the test measurements (less than $0.3 \%$ per hour).

A measure for the response fluctuations with time is given by a projection onto the ordinate and a subsequent fit with a Gaussian shape. Fluctuations, defined as (Gaussian) $\sigma /$ mean, larger than 0.2 are unacceptable. In total, $3.6 \%$ of the tubes exhibit a value larger than 0.2 (Figure 5 , bottom).

The dark current measurements, performed in a small dark box, resulted in a total of 20 R5505 tubes showing a dark current larger than $10 \mathrm{nA}$ (Figure 6). 

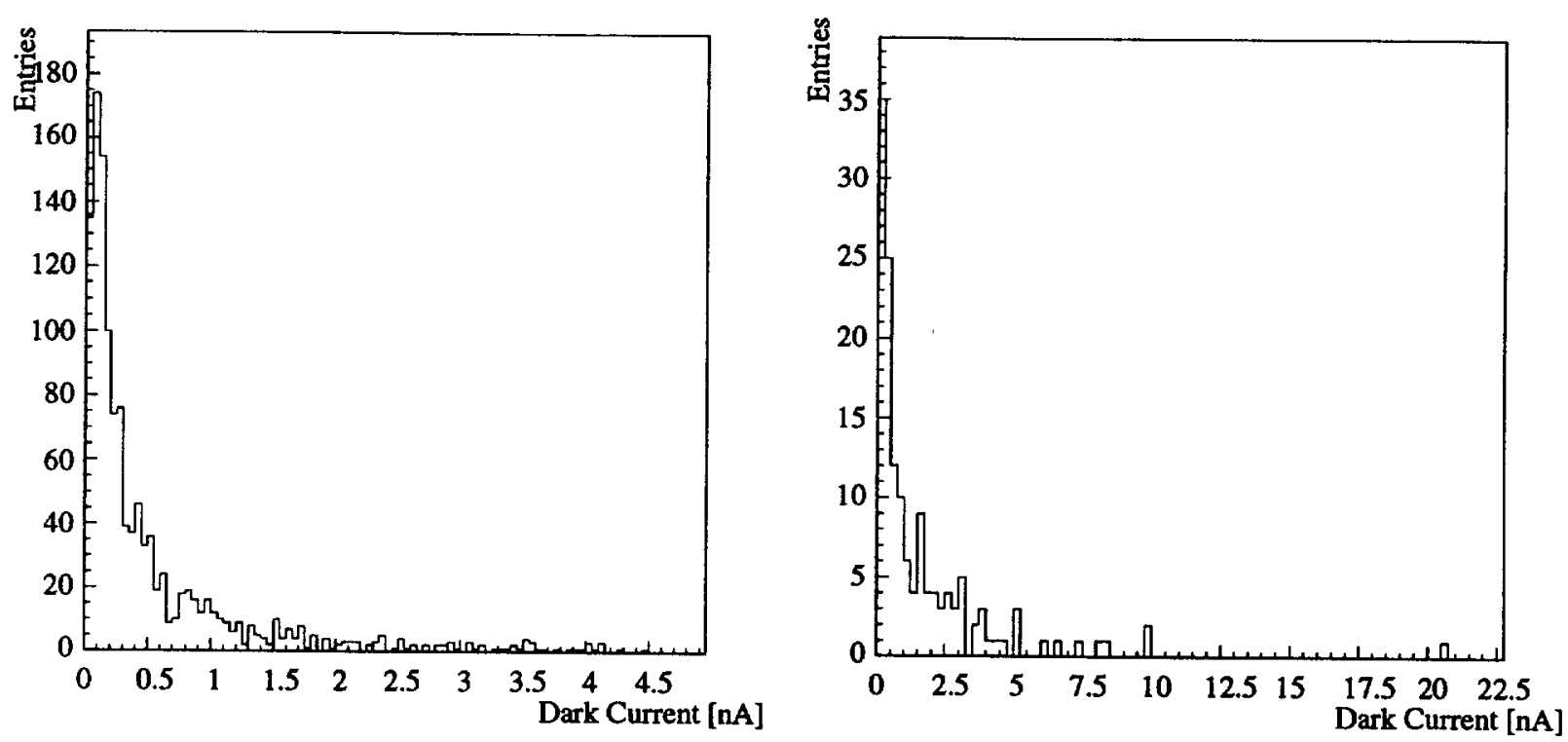

Figure 6: Left: Dark currents at $20^{\circ} \mathrm{C}$ for all $1^{\prime \prime}$ tubes. Right: Dark current distribution for the 2" tubes. According to [3] the dark current amplitudes double with every $5^{\circ} \mathrm{C}$ temperature increase.
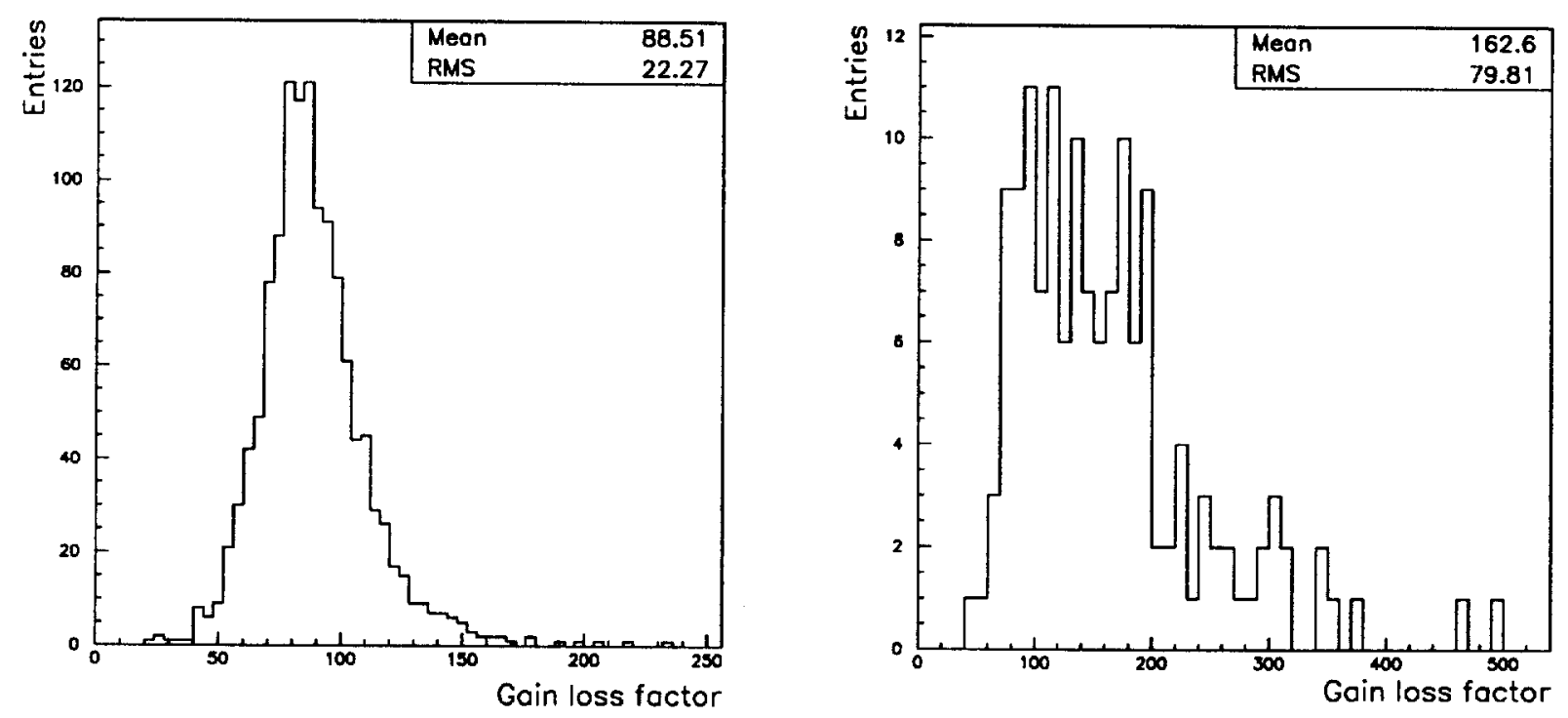

Figure 7: Gain loss factor at $1.2 T$ with respect to $0 T$. Left: Distribution for the 1 " tubes. Right: Distribution for the $2 "$ tubes.

\subsection{Results of Tests in a Magnetic Field}

In a previous series of test measurements with Hammatsu R5506 tubes it was observed [8] that the effective cathode area shrinks under the influence of a high magnetic field. Determination 
of the max-to-min ratio was therefore restricted to the cathode area inside a circle of $15 \mathrm{~mm}$ diameter. Another general feature is the appearance of a more pronounced central dip. This results in a higher max-to-min value measured at $1.2 \mathrm{~T}$ (see Figure 4, top). The mean value is now 1.6 (compared to 1.4 with no field). The effect of the magnetic field leads to another 14 tubes with max-to-min ratios larger than 2.5 which were rejected.

The gain loss caused by the magnetic field is determined by comparing measurements with and without field. A "gain loss factor" is defined as the ratio of phototube response with and without field. The absolute gain with field is then derived by multiplying the gain as given by the Hamamatsu data sheet (at $2000 \mathrm{~V}$ and $0 \mathrm{~T}$ ) by the gain loss factor from our tests. The distribution of the gain loss factors at $1.2 \mathrm{~T}$ for the R5505 tubes is shown in Figure 7 (left). The average gain loss factor at $1.2 \mathrm{~T}$ compared to no field amounts to 88 , with an r.m.s. spread as large as 22. This loss factor is valid when the tube axis is aligned in parallel to the field. The typical magnetic field inside the detector $\mathrm{H} 1$, at the position where the 1 " tubes are operated, is $0.95 \mathrm{~T}$. With this field the mean gain loss factor is only 25 , with an r.m.s. spread of 6 . The average absolute gain at $1.2 \mathrm{~T}$ is $1.2 \cdot 10^{4}$.

For the 2" tubes, the gain loss factor is even higher (Figure 7, right): At $1.2 \mathrm{~T}$ the average loss factor amounts to 163 , with a rather large r.m.s. spread of 80 . The field in $\mathrm{H} 1$ where these tubes operate is $0.88 \mathrm{~T}$ at the position of the hadronic SpaCal section. Here the gain loss factor is only 32, with an r.m.s. spread of 11 . The average absolute gain at $1.2 \mathrm{~T}$ for the 2 " tubes is $3.3 \cdot 10^{4}$, due to the higher amplification (16 instead of 15 stages). Table 2 gives an overview of the percentages of tubes which did not meet the specifications.

\begin{tabular}{|l|c|c|}
\hline Specification criterion & R 5505 & R 2490-06 \\
\hline \hline Max-to-min ratio $>2.5$ at 0 T & 2.2 & 8.3 \\
\hline Max-to-min ratio $>2.5$ at $1.2 \mathrm{~T}$ & 1.0 & - \\
\hline Dark Current $>10 \mathrm{nA}$ at $20^{\circ} \mathrm{C}$ & 1.4 & 0.7 \\
\hline Gain at $1.2 \mathrm{~T}<10^{4}\left[2^{\prime \prime}: 2 \cdot 10^{4}\right]$ & 46.8 & 20.1 \\
\hline
\end{tabular}

Table 2: Summary of specification tests: the numbers shown are the percentages of tubes which do not fulfil the specification. Tubes with a max-to-min ratio larger than 2.5 at $0 T$ have not been measured in the magnetic field; therefore the max-to-min criterion at $1.2 T$ delivers an additional percentage of tubes. The max-to-min ratio for $2^{\prime \prime}$ tubes at $1.2 T$ was not measured. For a discussion of the gain values see text.

\section{Test Measurements with a Selection of Tubes}

In the context of the series tests described above, a couple of measurements were made with a subset of phototubes in order to obtain a deeper understanding of the mechanisms determining their performance, and to find the limits of operation. We investigated the reactions of a small number of tubes to substantial and rapid changes of the environment temperature; we measured the change of gain as a function of the magnetic field varied in small steps between $0 \mathrm{~T}$ and 1.2 $\mathrm{T}$; we investigated the phenomenon of spontaneous high voltage discharges ("sparking"), measured the single photo-electron response of an R5505 tube, and finally tested the linearity of gain over a large dynamical range. 


\subsection{Phototube Response under the Influence of Temperature Changes}

In the running experiment, the phototubes have to operate in an environment with a temperature of $(32 \pm 2)^{\circ} \mathrm{C}$ (electromagnetic section) and $(20 \pm 2)^{\circ} \mathrm{C}$ (hadronic section). Depending on the number of power consuming devices switched on or off in the $\mathrm{H} 1$ detector, this temperature will undergo changes of some ${ }^{\circ} \mathrm{C}$ which might influence the gain of the tubes.

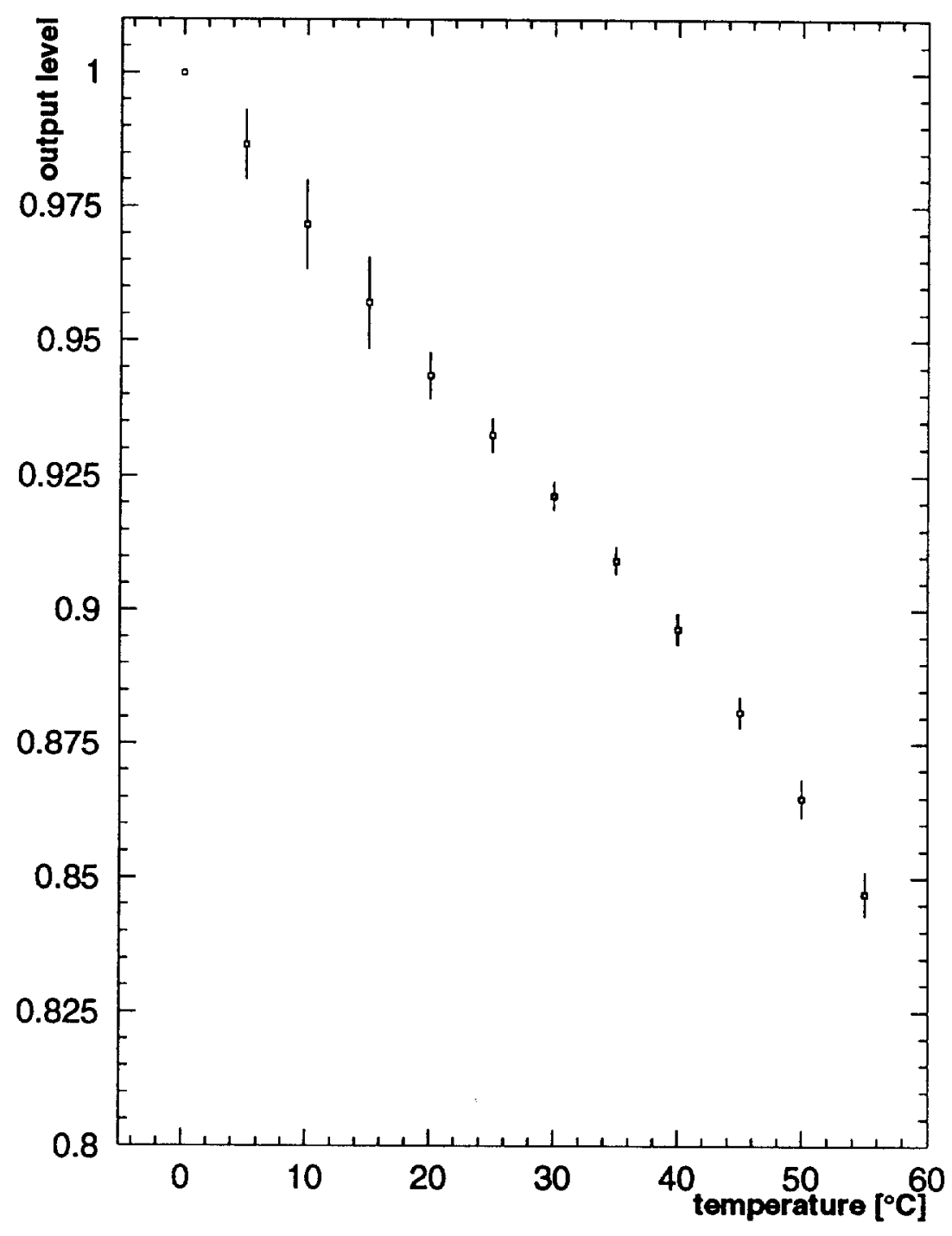

Figure 8: Reduction of the normalized output signal as a function of temperature, after stabilization (see text). Error bars indicate variations between individual tubes. 
We have therefore studied the temperature dependence of gains under well controlled conditions, using a programmable precise temperature chamber which provides a definite temperature of the tube's environment (air) [6]. The behaviour of four randomly selected tubes was investigated in a range between $0^{\circ} \mathrm{C}$ and $55^{\circ} \mathrm{C}$ in steps of $5^{\circ} \mathrm{C}$. The tubes were exposed to a constant temperature for several hours. The gain drop after temperature changes which proceed with a velocity of $\approx 2^{\circ} \mathrm{C} / \mathrm{min}$ takes place rather quickly, within several minutes. The new gain level is reached asymptotically after an "adaptation time" (see below) of the order of half an hour. The result, for a series of measurements between $0^{\circ} \mathrm{C}$ and $55^{\circ} \mathrm{C}$, with subsequent steps of $+5^{\circ} \mathrm{C}$, is shown in Figure 8: the data are normalized to the output value at $0^{\circ} \mathrm{C}$. Up to $20^{\circ} \mathrm{C}$ the output signal decreases with a constant slope of $-0.2 \% /{ }^{\circ} \mathrm{C}$, thereafter the slope increases to $-0.4 \% /{ }^{\circ} \mathrm{C}$.

After each temperature change, the time needed by the tubes to recover a stable output level was measured. This recovering process is well described by an exponential whose time constant is called "adaptation time". The distribution of these adaptation times is displayed in Figure 9. Adaptation after a temperature change is followed by a slight linear drift: the output level a few hours after the temperature change is somewhat different from that one immediately after the change. At lower temperatures, these drifts have a positive slope, i.e. recovery is accompanied by a slight increase of gain, while at higher temperature the drift becomes negative. Figure 10 shows an example for a typical drift behaviour.

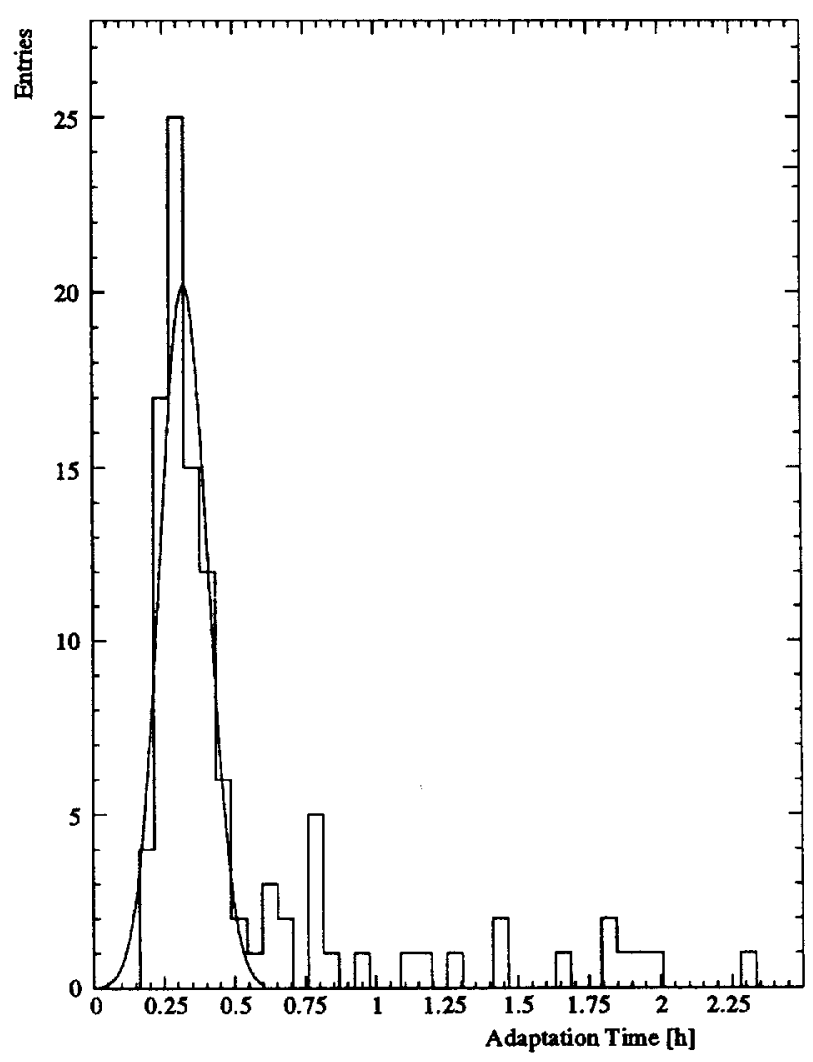

Figure 9: Distribution of adaptation times after temperature changes in steps of $5^{\circ} C$. 


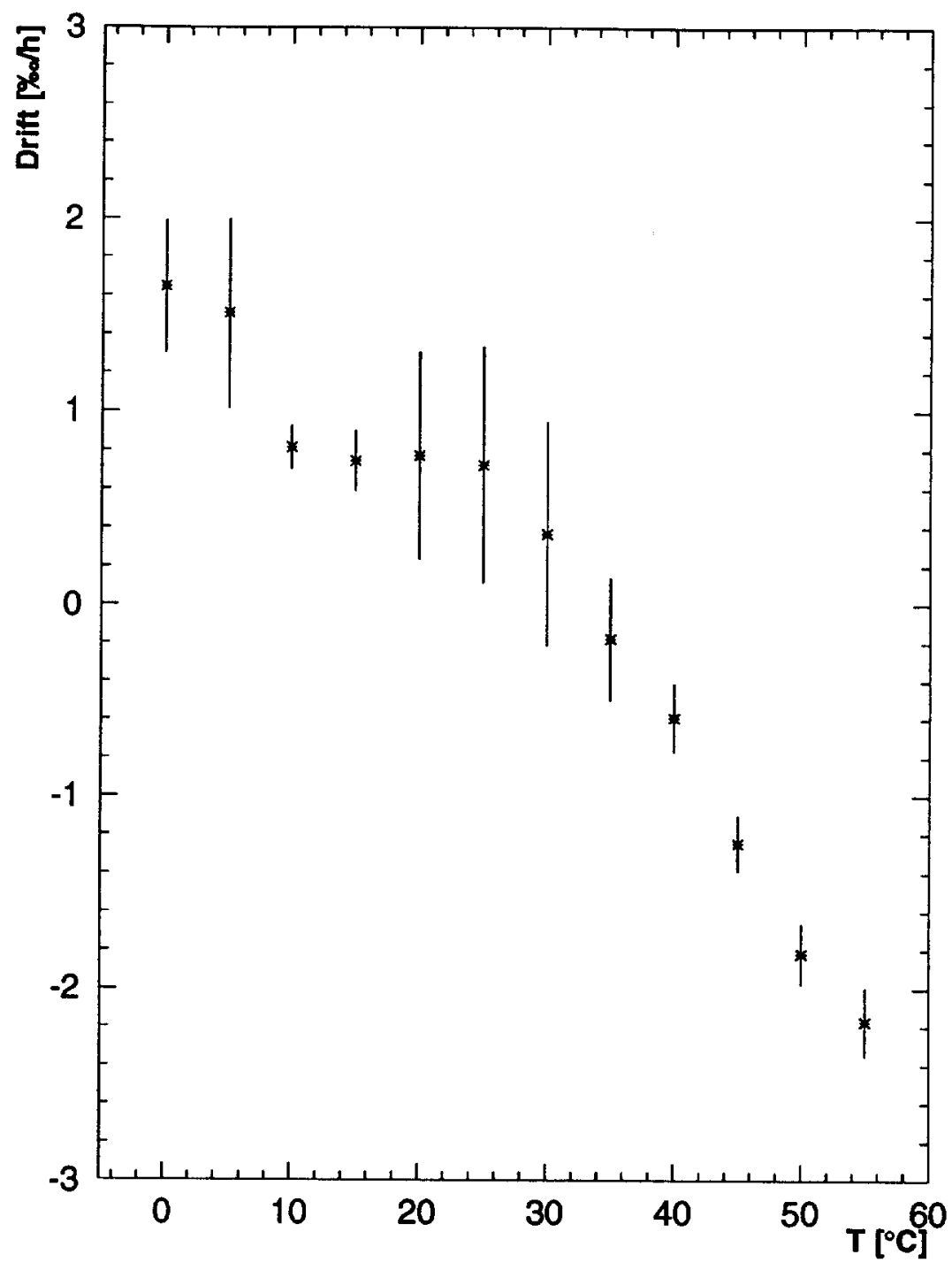

Figure 10: Distribution of drift values after temperature changes and subsequent adaptation in steps of $5^{\circ}$ C. Error bars indicate variations between individual tubes. 


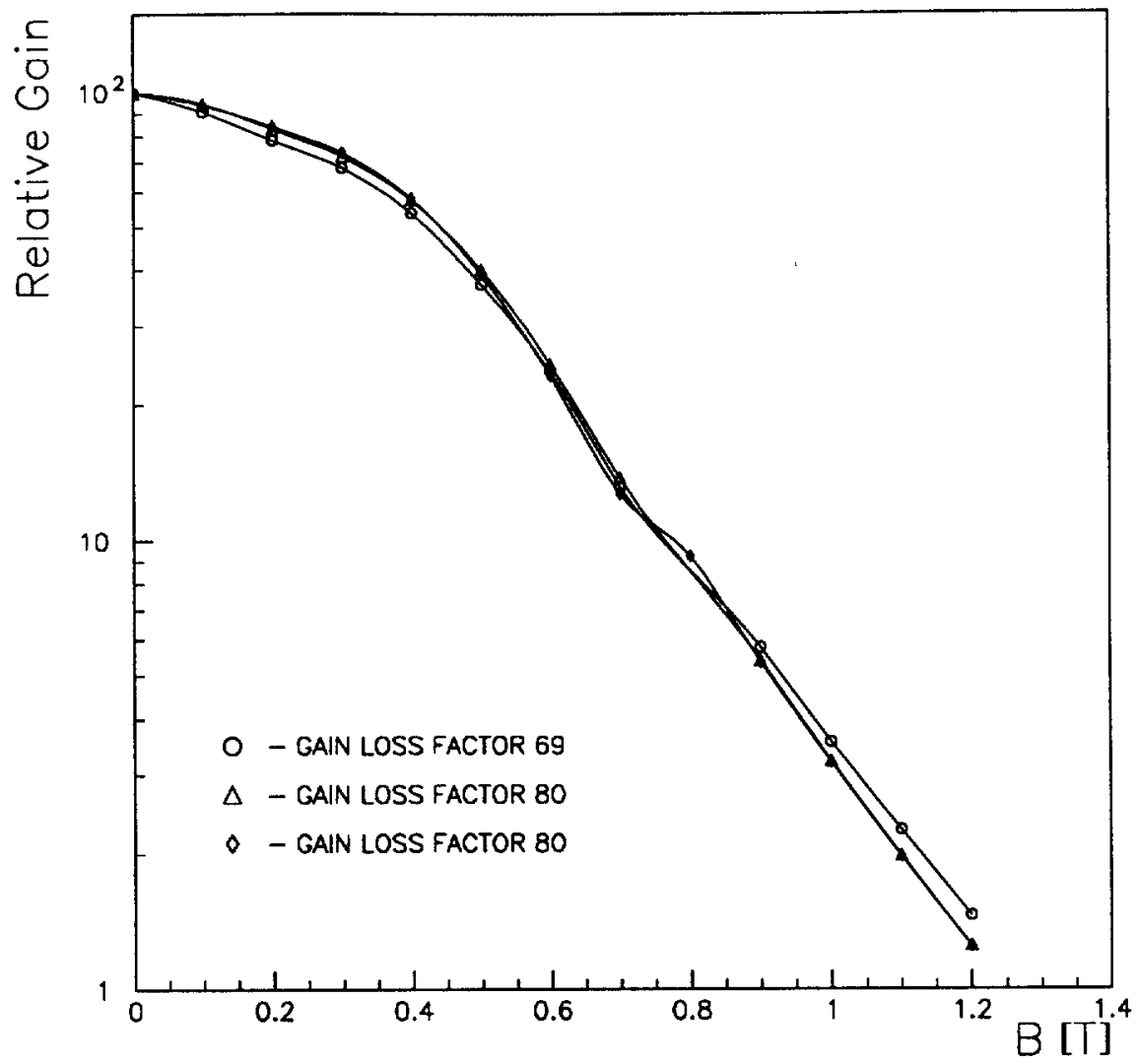

Figure 11: The normalized relative gain as a function of the magnetic field strength for three 1" tubes: after a rather modest decrease, an exponential drop starts around $0.4 T$. The magnetic field is aligned in parallel with the tube axis. 


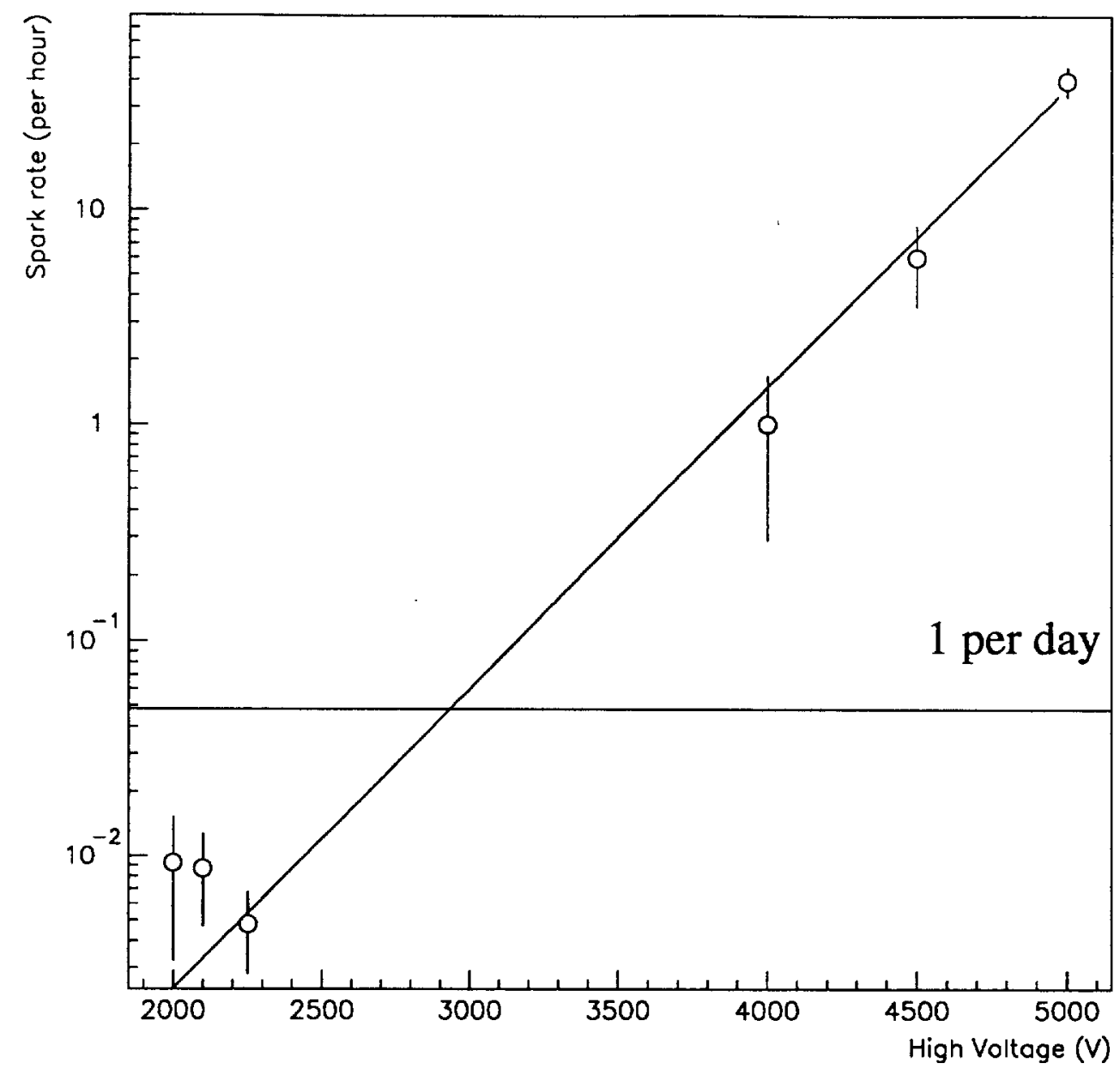

Figure 12: Spark rate as a function of high voltage. Measurements with $H V$ greater than $2.5 \mathrm{kV}$ have been performed with one single tube. No sparks were observed below $1900 \mathrm{~V}$. The constant line indicates a rate of one discharge per 24 hours.

\subsection{Gain Variation as a Function of the Magnetic Field}

The dependence of gain on the magnetic field was studied in detail with three tubes. The field was varied between $0 \mathrm{~T}$ and $1.2 \mathrm{~T}$ in steps of $0.1 \mathrm{~T}$. Figure 11 shows the relative gain normalized to an arbitrary value of 100 at $0 \mathrm{~T}$. After a rather modest decrease for low fields up to about 0.4 $T$, the gain starts dropping exponentially with $B$. The shape of the gain behaviour as a function of the magnetic field is in agreement with previous measurements and Monte-Carlo studies [5]. 

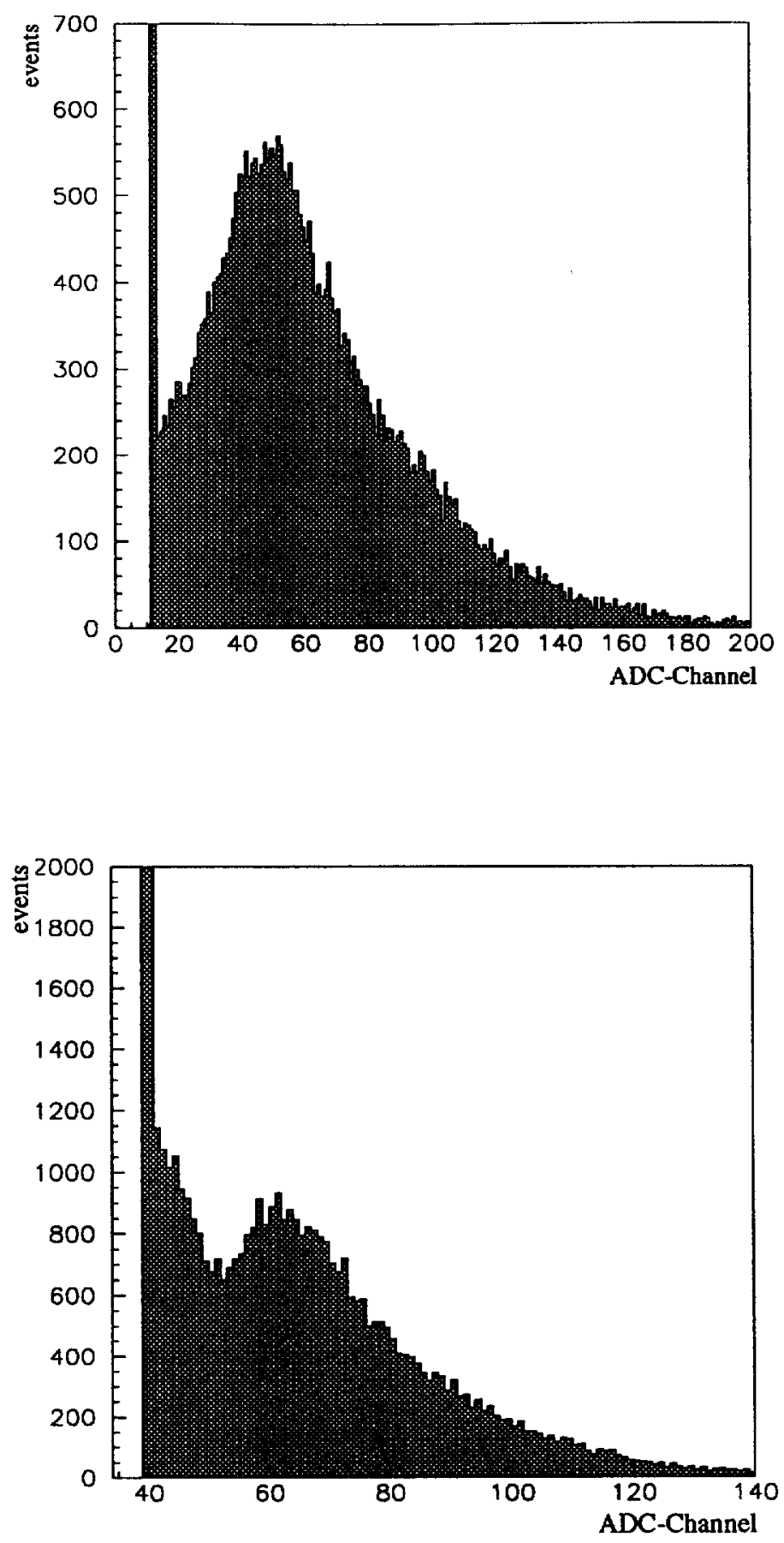

Figure 13: Single electron spectrum for a conventional photomultiplier tube (Philips XP 2008, top), and for a fine mesh tube (bottom). Here, the single electron peak is less clearly separated from the noise as roughly $45 \%$ of electrons miss the first dynode which leads to a lower gain. 


\subsection{Spontaneous High Voltage Discharges ("Sparking")}

During the homogeneity measurements it was noted that several ADC channels were damaged - mostly by destroying the $34 \Omega$ input resistors. These resistors, coupled to two diodes, serve as protections against excessive voltages (The specification is $\pm 50 \mathrm{~V}$ for a $1 \mu$ s duration, but the ADCs survive even $600 \mathrm{~V}$ for $1 \mu \mathrm{s}$ ). We conclude from the disruption that for a period of at least some microseconds a rather high voltage pulse was fed to the ADC input. To investigate this effect we coupled the output signals of 30 phototubes onto a panel with $50 \Omega$ resistors. After some time we looked for destroyed resistors.

Any effect of the environment (metal rack, light conductor, voltage divider etc.) could be excluded by systematic studies.

All observations led to the conclusion that vacuum breakthrough inside the tube is responsible for the destruction of the resistors.

Next, we studied the HV dependence of the effect with a single phototube. Figure 12 shows the spark rate as a function of $\mathrm{HV}$, where the points below $2.3 \mathrm{kV}$ result from the above mentioned measurements with 30 tubes. No destruction of resistors was observed below 1900 $\mathrm{V}$. Above $1900 \mathrm{~V}$ the spark rate $\mathrm{R}$ as a function of the high voltage $U$ can be fit by

$$
R(U)=1.6 \cdot 10^{-6} \exp \left(3.4 \cdot 10^{-3} U\right) \text { per hour, for } \mathrm{U}>1900 \mathrm{~V}
$$

The reason for the discharges is field emission of electrons inside the tubes, in particular from the sharp edges of the thin metal rods which connect the dynode grids. The light produced in the discharges could be observed directly in a separate measurement where two phototubes in coincidence were used to "look at" the sparks from a third one. As a solution for the SpaCal, a $2 \mathrm{M} \Omega$ resistor was inserted in the voltage divider between the high voltage connector and the cathode in order to limit the electrical energy available for a breakthrough such that no damage occurs.

\subsection{Response to Single Photo-Electrons}

The single photo-electron peak of an R5505 tube was measured using the LED pulser system as described above. By applying very short pulses at a large distance from the tube, the occurrence of non - single photo-electron pulses was drastically reduced according to a Poissonian probability density. First, the single photo-electron spectrum of a Philips XP 2008 tube was measured (Figure 13, top) for normalization. The single photo-electron peak is clearly visible. Next we illuminated one of the R5505 tubes with a very high gain $\left(4 \cdot 10^{6}\right.$ at $\left.2000 \mathrm{~V}\right)$. The tube was operated at $2500 \mathrm{~V}$, and the resulting spectrum is shown in Figure 13, bottom. The single electron peak is clearly distinguished, but the separation from the noise tail is not as good as for the conventional tube. The area between the pedestal and the single electron peak contains the electrons which missed the first dynode and therefore experienced a lower amplification. This suggestion is supported by the geometrical argument shortly discussed in section 2, and theoretical studies as described in [9]. From the single electron response the absolute gain can be determined. However, some care has to be taken in evaluating the gain: the common definition of gain is the ratio of anode to cathode current measured. In contrast to this definition, the gain value determined from the single photo-electron peak is based on the measured anode current (or charge) alone, and comes out by a factor of roughly 2.5 larger than the quoted Hamamatsu value. This can be understood by geometrical arguments: with the cathode current, one measures directly all the electrons emitted from the photocathode. Using the anode information alone, one loses all electrons which, due to the geometrical transmission 
factor of only about 0.4, do not reach the anode and thus do not contribute to anode current and amplification. The effective ratio of anode to cathode current becomes larger by a factor of roughly $1 / 0.4$, resulting in an apparently higher gain.

\subsection{Linearity of Gain}

Another property of interest is linearity. Detailed linearity tests undertaken at DESY in the summer of 1993 indicate that the Hamamatsu R5505 photomultiplier tubes used in the SpaCal calorimeter have excellent linearity properties. Linearity as used here refers to the proportionality between LED light input and output pulse heights at fixed HV. The tests were made by varying the distance between a fixed phototube and a moveable LED. The LED was pulsed by an external pulse generator so as to give in succession a large pulse (L), a small pulse (S), and no pulse $(P)$. The pulse duration was $100 \mathrm{~ns}$. This sequence could be repeated at various rates ranging from $10 \mathrm{~Hz}$ to $1000 \mathrm{~Hz}$. An ADC was used to digitize theses signals. The digitized (L-P) /(S-P) ratio was adjusted to a nominal fixed value close to 4 , and was very stable for the duration of each linearity measurement. Nonlinearities were expected to manifest themselves at high pulse heights when the phototubes start to saturate and the observed (L-P)/(S-P) ratio starts to decrease from its nominal value. Measurements made on four R5505 tubes, at various high voltages, both in a magnetic field of 1.2 Tesla and with no field, showed no deviations from linearity greater than $\pm 0.35 \%$ over a dynamic range of almost 1000 , up to anode currents of 100 $\mathrm{mA}(5$ Volt output pulses across $50 \Omega$ ) with $B=1.2 \mathrm{~T}$ and $300 \mathrm{~mA}(15$ Volt pulses across $50 \Omega$ ) with $B=0 \mathrm{~T}$. These were the highest pulse heights attainable with the equipment at hand. The value of $\pm 0.35 \%$ is an upper limit imposed by the stability of the pulse generator and the calibration of the attenuators used in these measurements. Thus, the linearity properties of these tubes may actually be much better than the limit reported here. It is worth noting that similar measurements with photomultiplier tubes having "squirrel cage" and in-line dynode structures showed much poorer linearity characteristics at high anode currents [10] than the mesh-dynode tube R5505 used in SpaCal.

\section{Summary of Experience after One Year of Opera- tion in $\mathrm{H} 1$}

\subsection{Commissioning of the Fine Mesh Phototubes}

The electromagnetic section of the SpaCal uses 1192 photomultiplier tubes with a 1" cathode diameter. In total, 1160 tubes of the type R5505 have been installed. Since the region near the beam pipe suffers from a higher radiation level of up to $50 \mathrm{~Gy} /$ year, the 32 tubes closest to the beam were chosen of the type R5506 with radiation hard UV glass windows.

In the hadronic section of the SpaCal, 128 phototubes of the type R2490-06 with a cathode diameter of 2 " were installed. In 1996, another 8 tubes of this type were added to some cells close to the outer circumference of the calorimeter.

Groups of 16 phototubes, i.e. the equipment of one supermodule, obtain their high voltage from a common HV board. Before installation, the photomultipliers were selected in such groups of 16 tubes of nearly equal gain at a common high voltage $U_{G}$, for a magnetic field strength of $0.95 \mathrm{~T}[0.88 \mathrm{~T}]$. The grouping of the PMTs was based on extrapolations from the test measurements described above. Individual high voltages in these groups can be adjusted within a range of $U_{G} \pm 160 \mathrm{~V}$. Keeping the photomultipliers at nearly equal gain is essential 
for equal trigger thresholds all over the calorimeter. Subsequent sizeable changes in gain lead to a serious dispersion of thresholds and have to be reduced by modifying the individual high voltages. Smaller changes are monitored by a LED calibration system [11] (see below) and corrected in the offline calibration procedure.

After installing the phototubes and a short "burning in" phase, all calorimeter channels were tested with cosmic muons penetrating several calorimeter cells at a time, and the phototube gain equalized by adjusting the high voltage appropriately. During these tests, three R5505 tubes were found dead due to problems with the vacuum tightness of the socket pins, and were exchanged. Another four tubes showed gains much smaller than determined before. These tubes were also exchanged and sent back to the manufacturer.

Finally, the calorimeter was installed in the $\mathrm{H} 1$ pit without any further possibility for access to the phototubes for a period of about 10 months. During the first four months, high voltage for the tubes was frequently switched off during electron and proton beam injections in order to protect them from the enhanced radiation level. In addition, due to the high power consumption in $\mathrm{H} 1$ and the heating effect of the beam radiation, the temperature at the SpaCal went up to as much as $40^{\circ} \mathrm{C}$ during operation, as measured outside the phototube housings. At that temperature the high voltage was switched off in order to prevent photocathode damage and to allow the tubes to cool down to about $32^{\circ} \mathrm{C}$. This was done twice a week on the average. After some weeks, the water cooling of the calorimeter was improved, and the maximum temperatures measured levelled off at $\approx 35^{\circ} \mathrm{C}$. For the last three months, the high voltages were kept at the selected operation values even during injection of the HERA beams. All results presented below refer to this period of stable high voltage operation, with temperature changes of less than $5^{\circ} \mathrm{C}$.

No further tube was found broken during this time. Only three tubes showed a decrease in gain too large to be compensated by raising the high voltage.

\subsection{Monitoring the Phototube Gain Variations}

The phototube gains were permanently monitored with the pulsed light of LEDs (Toshiba TLGP 240 [13]). Their signals were sent via $1 \mathrm{~mm}$ thin flexible light guiding fibres to plexiglass light mixers glued onto the surfaces of the photocathodes. Fluctuations of the light emission of the LEDs were recognized by photodiodes directly coupled to them. The photodiodes (Siemens $\mathrm{SFH} 2030$ ) have a temperature coefficient of $0.18 \% /{ }^{\circ} \mathrm{C}$. The temperature in the detector was permanently monitored with a precision of better than $0.3^{\circ} \mathrm{C}$. In this way the temperature dependence of the LEDs could be unfolded, and the signal response of the phototubes could be corrected for gain changes due to temparature variations, long time gain drifts, or ageing.

Two examples for the gain behaviour of individual phototubes are presented in Figure 14. Figure 14 (top) shows, for a standard R5505 phototube, the signal normalized to the photodiode signal over a time interval of 38 hours. The gain variations are large, up to $\pm 15 \%$. Figure 14 (bottom) shows, for the same period, the corresponding distribution for a neighbouring tube in the same calorimeter module. Here, variations are much smaller, below $1 \%$, and can be related to small temperature or high voltage fluctuations. No correlation in gain variation with the other tube is observed.

A global description of the gain variations of all phototubes in both in the electromagnetic and in the hadronic calorimeter section can be read from Figures 15 and 16. Figure 15 shows the normalized gain and the $\pm 1 \sigma$ contours for the phototubes in the electromagnetic section of the calorimeter, and two projections onto the ordinate, one at the very beginning of the data taking period, and one 19 days later. The widening of the distribution of individual gains proceeds rather fast for an interval of five days and flattens thereafter. The few spikes observed 
are produced partially by temperature bumps or corrupted calibration events. About $80 \%$ of all tubes have gain changes of less than $2 \%$ [11] within the time quoted. Only 12 PMTs have gain variations larger than $\mathbf{1 0 \%}$. The corresponding plot for the tubes in the hadronic section is shown in Figure 16.

These gain changes can be corrected for by using the photodiode signals and the temperature measurements inside the detector. After these corrections, the mean value of the reference energy from electrons scattered into the "kinematic peak" region is found to be rather stable, with deviations below $1 \%$ [12].
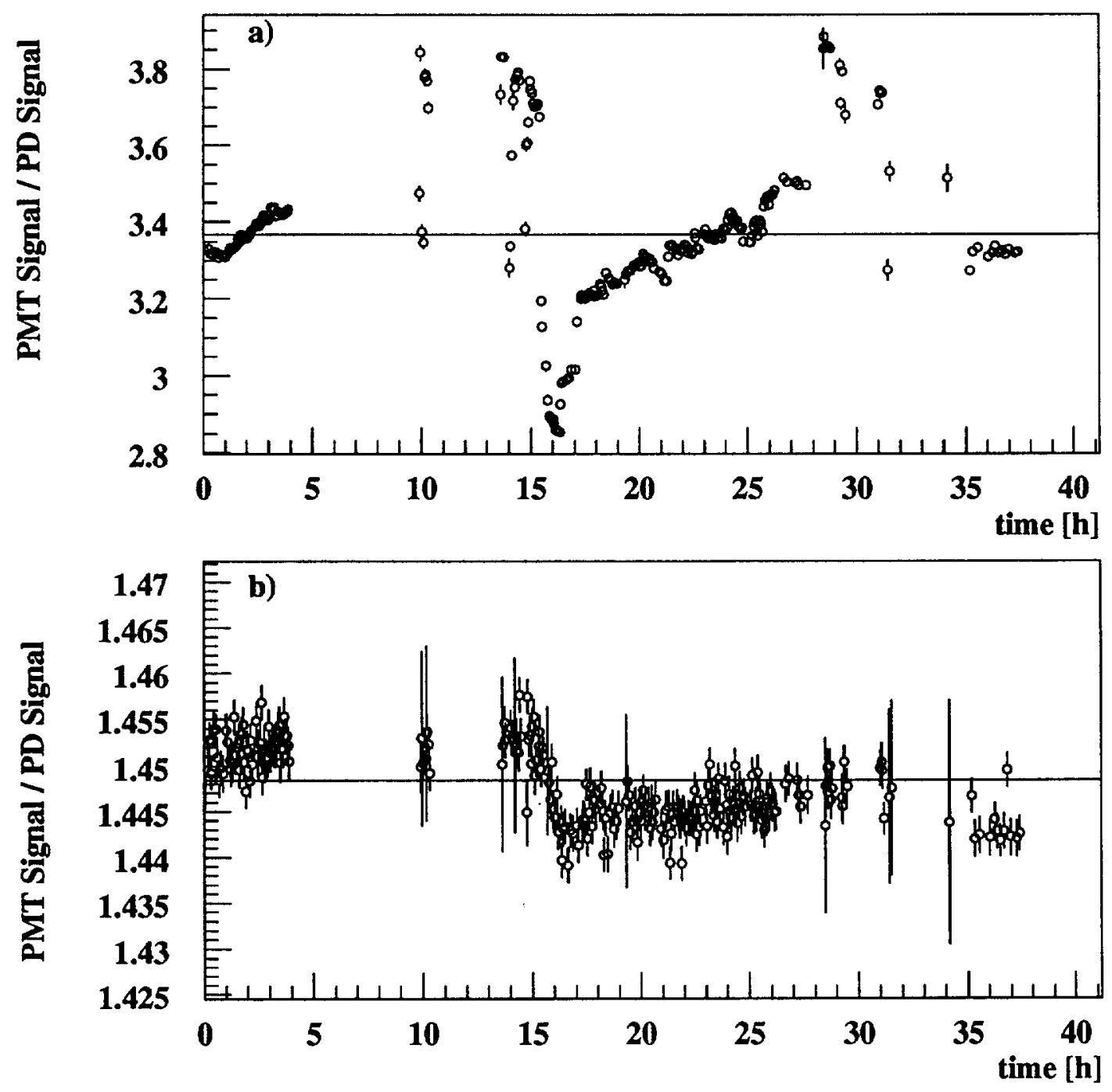

Figure 14: Gain change of two selected tubes over a period of 38 hours. The plots show the ratios of phototube to photodiode signals. 

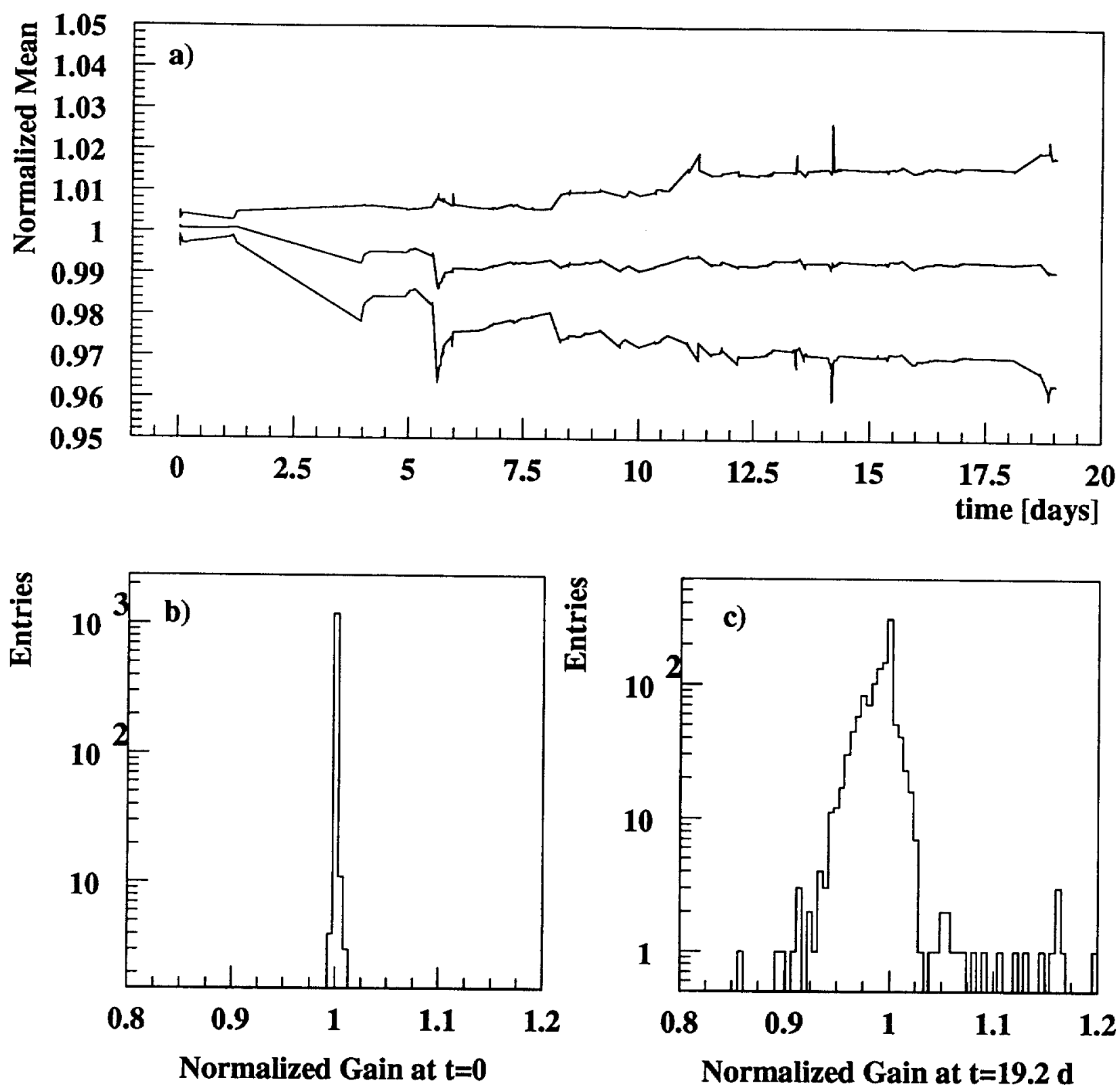

Figure 15: a) Long term gain development for all tubes in the electromagnetic section: normalized mean gain and $\pm 1 \sigma$ contours. The lower plots show two projections, one (b) at the very beginning and one (c) at the end of the running period. 

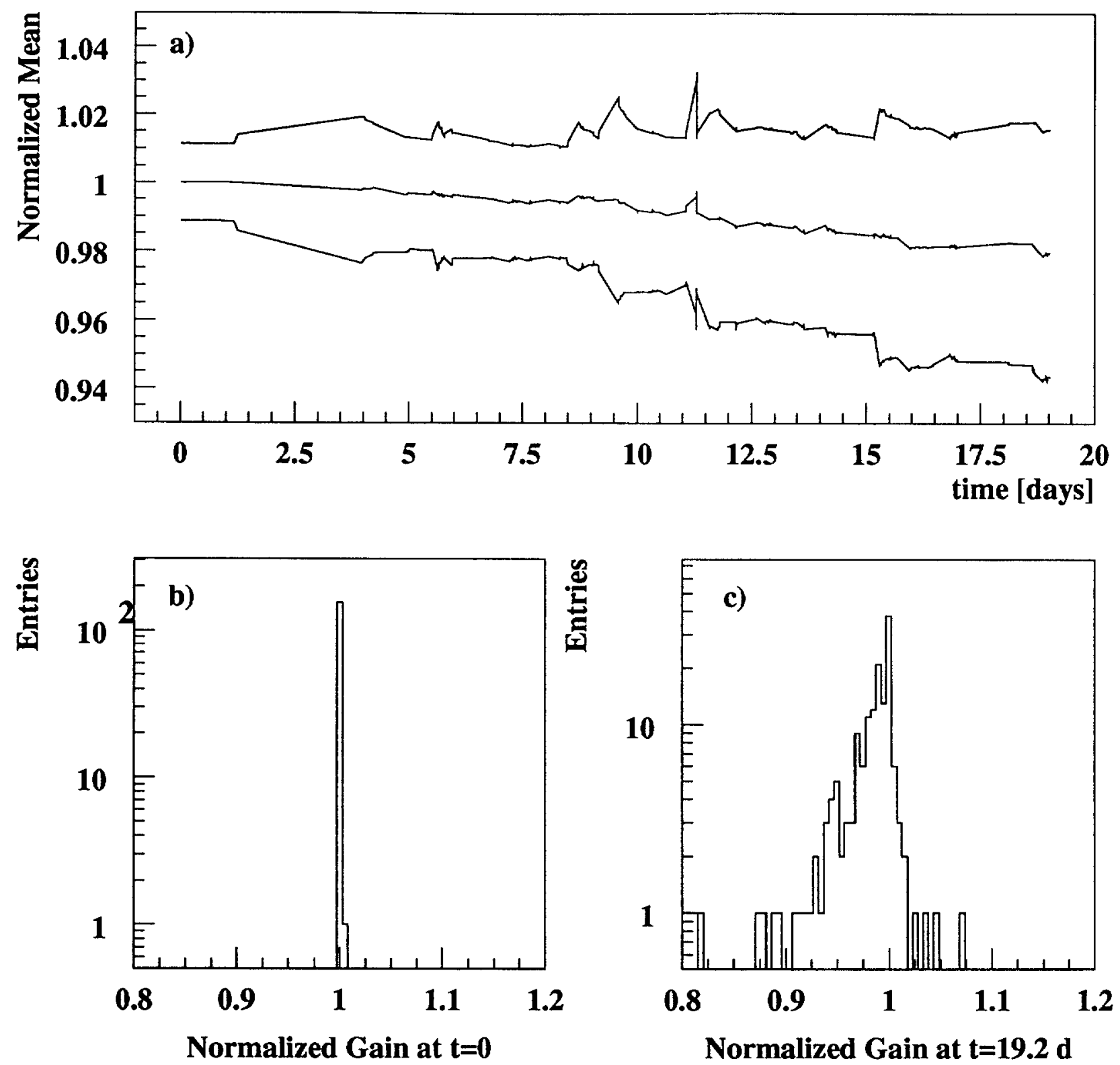

Figure 16: a) Long term gain development for all tubes in the hadronic section: normalized mean gain and $\pm 1 \sigma$ contours. The lower plots show two projections, one (b) at the very beginning and one (c) at the end of the running period.

\section{Conclusions}

We have performed a series test on a large number of Hamamatsu fine mesh phototubes of types R5505 and R2490-06 for application in a strong magnetic field. The average gain loss factors between operation at $0 \mathrm{~T}$ and at $1.2 \mathrm{~T}$ have been determined to be 88 for the R5505, and 162 for the R2490-06 tube, with large ( $25 \%-50 \%$ ) spreads of the distributions of individual gain losses. The average absolute gains at $2000 \mathrm{~V}$ are $1.2 \cdot 10^{4}$ and $3.3 \cdot 10^{4}$, respectively. An inhomogeneity 
of the cathode response present at $0 \mathrm{~T}$ is slightly enhanced in a magnetic field of $1.2 \mathrm{~T}$. For a subsample of tubes, the response with respect to temperature changes has been investigated, and a fine step measurement of the gain as a function of a magnetic field between $0 \mathrm{~T}$ and 1.2 $\mathrm{T}$ has been performed. Occurrence of high voltage discharges was observed and explained as vacuum break-throughs due to field emission, and preventive measures for operation in the SpaCal were developed. The single photo-electron peak for an R5505 tube was measured which gives a handle for an independent determination of the absolute gain, and the range of gain linearity was investigated.

\section{Acknowledgements}

We gratefully acknowledge the assistence of the electronic and mechanical workshops of the Institut für Hochenergiephysik of the Universität Heidelberg in constructing the test setups, and the DESY MEA group for supplying the magnet and their support.

\section{References}

[1] J. Bán et al. (The H1 BEMC Group), Nucl. Instr. Meth. A372 (1996) 399

[2] T. Nicholls et al. (The $\mathrm{H} 1$ SpaCal Group), "Performance of an Electromagnetic Lead/Scintillating-Fibre Calorimeter for the H1 Detector", Nucl. Instr. Meth. A374 (1996) 149

R. D. Appuhn et al. (The H1 SpaCal Group), "Hadronic Response and e/ $\pi$ Separation with the H1 Lead/Scintillating-Fibre Calorimeter", Nucl. Instr. Methods A382 (1996) 395 R. D. Appuhn et al. (The H1 SpaCal Group), "The H1 Lead/Scintillating-Fibre Calorimeter", DESY 96-171 (1996) (submitted to Nucl. Instr. Meth. A)

[3] R. D. Appuhn et al. (The H1 SpaCal Group), "H1 backward upgrade with a SpaCal Calorimeter: The Hadronic Section", DESY 96-013 (1996)

[4] Hamamatsu Photonics Data Sheets

[5] J. Janoth et al., Nucl. Instr. Meth. A350 (1994) 221

[6] R. Schediwy, Diplomarbeit, Universität Heidelberg (1996)

[7] "Photomultipliers and Assemblies for Scintillator Counting and High Energy Physics", Hamamatsu , March 1993, "Photomultiplier Tubes (Principle and Application)", Hamamatsu 1994

[8] T. Bipp, Diplomarbeit, Universität Hamburg, 1995

[9] R. Enomoto et al., Nucl. Instr. Meth. A332 (1993) 129

[10] H. Steiner, Private Communication

[11] J. Janoth, Dr. rer. nat. Dissertation, Universität Heidelberg (1996)

[12] A. Meyer, Dr. rer. nat. Dissertation, Universität Hamburg, to be published 1997

[13] J. Janoth, Diplomarbeit, Universität Dortmund (1993) 
